\title{
Identification of Genomic Regions Controlling Adult-Plant Stripe Rust Resistance in Chinese Landrace Pingyuan 50 Through Bulked Segregant Analysis
}

\author{
Caixia Lan, Shanshan Liang, Xiangchun Zhou, Gang Zhou, Qinglin Lu, Xianchun Xia, and Zhonghu He
}

\begin{abstract}
First, second, sixth, and seventh authors: Institute of Crop Science, National Wheat Improvement Center/The National Key Facility for Crop Gene Resources and Genetic Improvement, Chinese Academy of Agricultural Sciences (CAAS), Zhongguancun South Street 12, Beijing 100081, China; second author: Department of Primary Industries, Victorian AgriBiosciences Center, La Trobe R\&D Park, 1 Park Drive, Bundoora, Vic 3083, Australia; third, fourth, and fifth authors: Gansu Wheat Research Institute, Gansu Academy of Agricultural Sciences, Lanzhou 730070, Gansu Province, China; and seventh author: International Maize and Wheat Improvement Center (CIMMYT), CIMMYT China Office, c/o CAAS, Zhongguancun South Street 12, Beijing 100081, China.
\end{abstract} Accepted for publication 16 November 2009.

\section{ABSTRACT}

Lan, C. X., Liang, S. S., Zhou, X. C., Zhou, G., Lu, Q. L., Xia, X. C., and He, Z. H. 2010. Identification of genomic regions controlling adult-plant stripe rust resistance in Chinese landrace Pingyuan 50 through bulked segregant analysis. Phytopathology 100:313-318.

Stripe rust, caused by Puccinia striiformis f. sp. tritici, is one of the most widespread and destructive wheat diseases worldwide. Growing resistant cultivars with adult-plant resistance (APR) is an effective approach for the control of the disease. In this study, 540 simple sequence repeat markers were screened to map quantitative trait loci (QTL) for APR to stripe rust in a doubled haploid (DH) population of 137 lines derived from the cross Pingyuan $50 \times$ Mingxian 169. The DH lines were planted in randomized complete blocks with three replicates in Gansu and Sichuan provinces during the 2005-06, 2006-07, and 2007-08 cropping seasons, providing data for four environments. Artificial inoculations were carried out in Gansu and Sichuan with the prevalent Chinese race CYR32. Broad-sense heritability of resistance to stripe rust for maximum disease severity was 0.91 , based on the mean value averaged across four environments. Inclusive composite interval mapping detected three QTL for APR to stripe rust on chromosomes 2BS, 5AL, and 6BS, designated QYr.caas-2BS, QYr.caas-5AL, and QYr.caas-6BS, respectively, separately explaining from 4.5 to $19.9 \%$ of the phenotypic variation. QYr.caas-5AL, different from QTL previously reported, was flanked by microsatellite markers Xwmc410 and Xbarc261, and accounted for 5.0 to $19.9 \%$ of phenotypic variance. Molecular markers closely linked to the QTL could be used in marker-assisted selection for APR to stripe rust in wheat breeding programs.

Additional keywords: durable resistance, microsatellites, Triticum aestivum.
Stripe rust, caused by Puccinia striiformis Westend. f. sp. tritici Eriks., is a devastating wheat disease worldwide, particularly in the areas where wheat is grown under cool and temperate conditions or at high altitudes (31). China is the largest stripe rust epidemic region in the world, and stripe rust is very destructive to autumn-sown wheat in northwestern and southwestern parts of the country, where weather conditions are conducive for disease development every year $(13,29)$. Resistant cultivars are the most efficient, economically viable, and environmentally friendly means of controlling the disease (16).

Due to frequent changes in the pathogen population, cultivars with major race-specific resistance genes lose their resistance to stripe rust within a few years of cultivation (27). As a consequence, adult-plant resistance (APR) with reputed durability is increasingly being used in wheat breeding and production $(14,17$, 23,30). APR is characterized by susceptibility at the seedling stage, but resistance at the adult stage when inoculated with the same pathotypes.

To date, 47 stripe rust resistance genes at 42 loci have been catalogued, and about 33 have been assigned temporary designations (18). Ten of them, viz. Yr11, Yr12, Yr13, Yrl4, Yrl6, Yr18, Yr29, Yr30, Yr36, and Yr39 are APR genes. The first four

Corresponding authors: X. C. Xia and Z. H. He

E-mail addresses: xiaxianchun@ caas.net.cn and zhhecaas@gmail.com

doi:10.1094/PHYTO-100-4-0313

(C) 2010 The American Phytopathological Society are poorly defined and there is evidence for virulence on plants with $\operatorname{Yrll}$ (9) and likely virulence for resistances attributed to Yr12, $Y r 13$, and $Y r 14$ from pathogenicity data published in reports by the National Institute of Agricultural Botany in the U.K. On the other hand, APR genes Yr16, Yr18, Yr29, Yr30, Yr36, Yr39 and others characterized only as QTL are considered to be sources or components of nonspecific durable resistances, although most individually do not confer adequate levels of protection and need to be deployed in combinations. Genetic studies on lines that have acceptable levels of APR usually indicate involvement of three or more such genes $(3,17,23)$. Therefore, it is very important to map APR genes and to identify closely linked molecular markers that are useful for combining APR genes in wheat breeding.

The Chinese wheat landrace Pingyuan 50 was an important cultivar in the Yellow and Huai Valleys of China in the 1950s (5). It was originated from the northern Henan province, and then spread to all parts of Henan and western part of Shandong province. Its area expanded rapidly after the stripe rust epidemic in 1950, and reached about 270,000 to 400,000 ha annually in the 1950s (5). The cultivar is known to have maintained a moderate level of resistance to all races of $P$. striiformis f. sp. tritici in the field for over 50 years $(32,33)$. The aim of this study was to identify and map QTL for APR to stripe rust in a doubled haploid (DH) population generated from the cross Pingyuan $50 \times$ Mingxian 169. Knowledge about the genetic basis of stripe rust resistance in Pingyuan 50 will enable more efficient selection of its resistance genes in wheat breeding programs. 


\section{MATERIALS AND METHODS}

Plant materials. The $137 \mathrm{DH}$ lines used in this study were developed from a cross between Chinese landrace cultivars Pingyuan 50 and Mingxian 169 by the wheat $\times$ maize crossing technique (11). Mingxian 169 is a landrace from Shanxi province, and is highly susceptible to all races of $P$. striiformis f. sp. tritici at all growth stages.

Field trials. The DH lines and their parents were evaluated for APR to stripe rust at Qingshui, Gansu province, and Chengdu, Sichuan province during the 2005-06, 2006-07, and 2007-08 cropping seasons. The weather conditions in these areas are considered to be very favorable for stripe rust infection and spread.
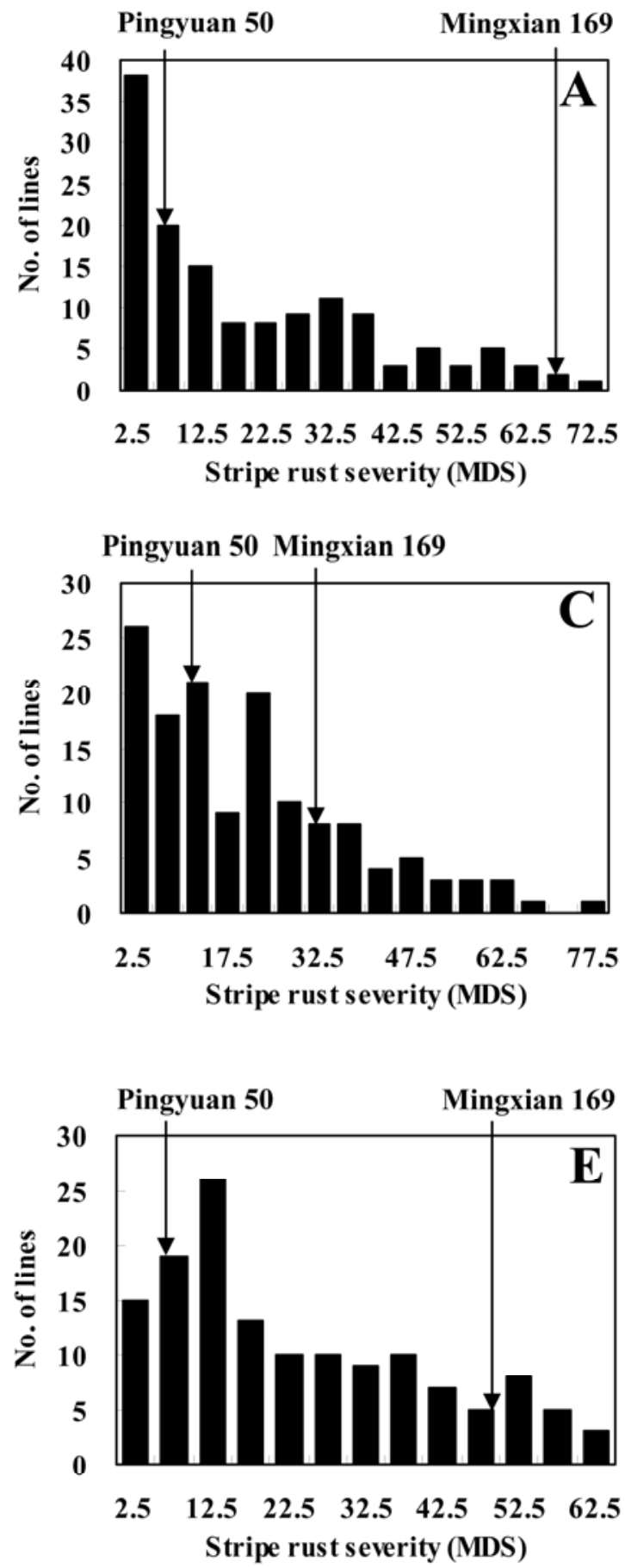

Field trials were conducted in randomized complete blocks with three replicates. Plots consisted of single rows of $1.5 \mathrm{~m}$ length and $25 \mathrm{~cm}$ between rows. Approximately 50 seeds were sown in each row.

Seeds were sown in autumn in Qingshui, Gansu province. Mingxian 169 was planted after every 10 rows as a susceptible check, and Huixianhong, which is also highly susceptible to stripe rust, was planted around the experimental area to ensure ample inoculum in spring. Inoculations were performed with race CYR32 at the three-leaf stage in spring. Stripe rust severities were assessed for the first time 4 weeks after inoculation, and then at weekly intervals on two further occasions using the modified Cobb scale (19).
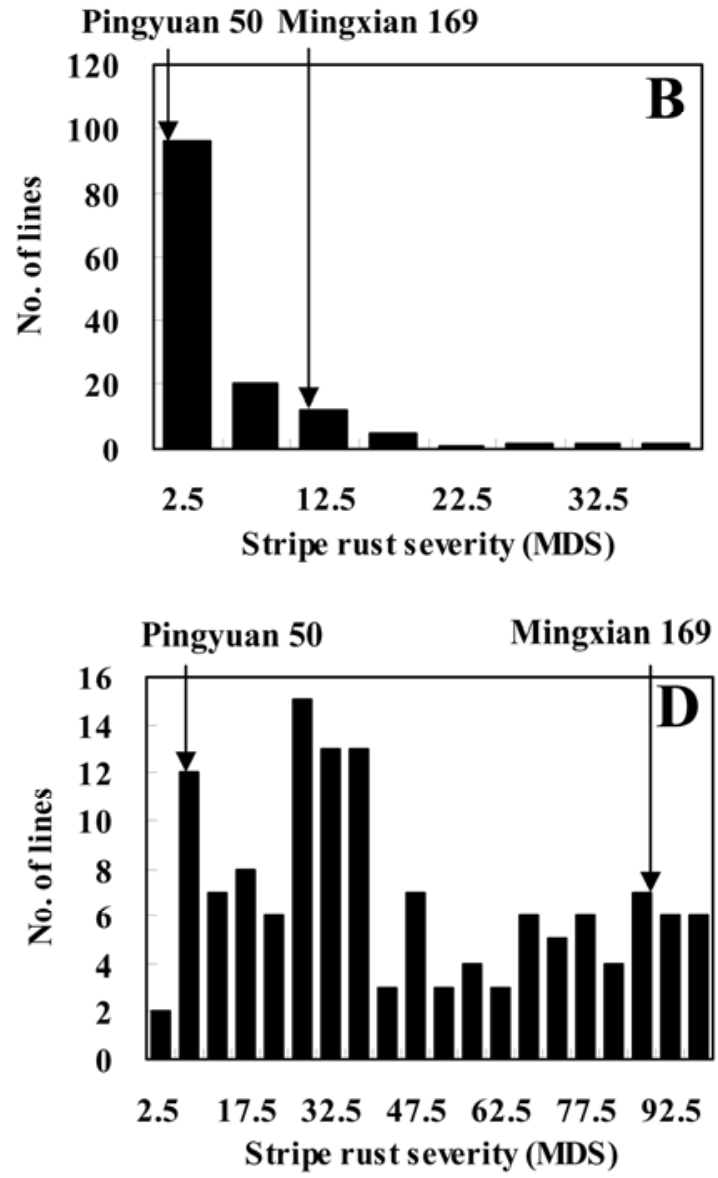
In the 2007-08 cropping season, the DH lines and their parents were sown in winter in Chengdu, Sichuan province. Mingxian 169 was planted after every 10 rows as a susceptible check, and Chuanyu 12 and Sy95-71, which are highly susceptible to stripe rust, were planted around the tested lines to ensure ample inoculum. Inoculation was also performed with race CYR32, the prevalent race in the region, at the three-leaf stage. Stripe rust severities were rated, when the disease severities on Mingxian 169 had reached a maximum level around 20 April 2008.

Statistical analysis. Maximum disease severity (MDS) was used for analysis of variance (ANOVA) and QTL detection. SAS software (SAS Institute, Cary, NC) was used to compute ANOVA. Broad-sense heritability $\left(h^{2}\right)$ estimates were based on the formula: $h^{2}=\sigma_{g}^{2} /\left(\sigma_{g}^{2}+\sigma_{g e}^{2} / e+\sigma_{\varepsilon}^{2} / r e\right)$, where $\sigma_{g}^{2}, \sigma_{g e}^{2}$, and $\sigma_{\varepsilon}^{2}$ are estimates of genotype, genotype $\times$ environment interaction, and error variances, respectively, and $e$ and $r$ are the numbers of environments and replications per environment, respectively.

Simple sequence repeat analysis. Of 540 pairs of wheat simple sequence repeat (SSR) primers, 273 pairs were wheat microsatellite consortium (WMC) primers (7), 160 pairs were Beltsville Agriculture Research Center (BARC) primers (25), 101 pairs were Gatersleben Wheat Microsatellite (GWM) primers (21), 4 pairs were Clermont Ferrand A-genome (CFA) primers (26), and 2 pairs were Clermont Ferrand D-genome (CFD) primers (8).

Bulked segregant analysis. Based on maximum disease severities, resistant and susceptible bulks were constructed by mixing equal amounts of DNA from the five most resistant and five most susceptible DH lines, respectively. SSRs showing polymorphisms between the two parents and between the two bulks were used to genotype all $137 \mathrm{DH}$ lines to determine their associations with stripe rust responses. Additional SSRs around

TABLE 1. Analysis of variance of maximum disease severity scores for the doubled haploid population derived from the cross Pingyuan $50 \times$ Mingxian 169

\begin{tabular}{lrrrr}
\hline Source of variation & \multicolumn{1}{c}{ df } & \multicolumn{1}{c}{$\begin{array}{c}\text { Sum of } \\
\text { squares }\end{array}$} & Mean square & $F$ value $^{\mathrm{a}}$ \\
\hline Replicate & 2 & $1,882.06$ & 941.03 & $11.70^{* *}$ \\
Environment & 3 & $404,739.73$ & $134,913.24$ & $1,677.92^{* *}$ \\
Line & 136 & $217,094.84$ & $1,596.29$ & $19.85^{* *}$ \\
Line $\times$ environment & 408 & $231,426.17$ & 571.42 & $7.11^{* *}$ \\
Error & 1,069 & $86,194.29$ & 80.41 & \\
\hline
\end{tabular}

a $* *$ indicates $P<0.0001$. the loci associated with APR were selected from available linkage maps (24) and those polymorphic were genotyped on the entire DH population.

Map construction and QTL detection. Mapmaker 3.0b (15) software was used to establish linkage groups. The mapping function of Kosambi (10) was used to convert recombination values to genetic distances. The software IciMapping 2.2 was used to detect QTL by the inclusive composite interval mapping (ICIM) method (12). A logarithm of odds (LOD) of 2.0 was set to declare QTL as significant. The percentages of phenotypic variance explained (PVE) by individual QTL and additive effects at the LOD peaks were obtained through stepwise regression (12). Each QTL was represented by a 20-centimorgan (cM) interval with the local LOD maximum at the central point. Adjacent QTL on the same chromosome were considered different when the distances between the curve peaks were greater than $20 \mathrm{cM}$ or when the support intervals were non-overlapping.

\section{RESULTS}

Phenotypic analysis of MDS, correlations, and heritabilities. The MDS data from four environments were significantly correlated $(P<0.0001)$, with the correlation coefficients ranging from 0.37 to 0.71 . The MDS frequency distribution indicated quantitative inheritance of APR to stripe rust (Fig. 1). The mean MDS of Pingyuan 50 and Mingxian 169 in Gansu were 5.7 and $66.7 \%, 0.3$ and $11.3 \%$, and 11.0 and $31.6 \%$ in 2006, 2007 , and 2008, respectively, whereas the means of the resistant and susceptible parents were 6 and 90\%, respectively, in Sichuan 2008. The average of MDS of the DH lines over four environments was $23.3 \%$, ranging from 0 to $100 \%$. The broad-sense heritability of MDS was 0.91 based on across-environment genotype means. ANOVA revealed significant variation among the DH lines (Table 1).

QTL analysis for APR to stripe rust. Based on the mean MDS in each environment and the means over four environments, three QTL for APR to stripe rust were detected on chromosomes 2BS, 5AL, and 6BS (Table 2; Fig. 2), and designated QYr.caas$2 B S, Q Y$ r.caas-5AL, and QYr.caas-6BS, respectively.

The QTL QYr.caas-2BS, detected on chromosome 2BS in the SSR marker interval Xbarc13-Xbarc230, was identified in Gansu 2006, 2007, and 2008, accounting for 5.1, 9.5, and 5.7\% of the phenotypic variance with additive effects of $-4.25,-2.30$, and -4.11, respectively. The most stable QTL, QYr.caas-5AL, was located between Xwmc410 and Xbarc261 on the long arm of chromosome 5A, explaining 6.2, 5.0, 19.9, and $16.5 \%$ of the

TABLE 2. Quantitative trait loci (QTLs) for maximum disease severity (MDS) to stripe rust by inclusive composition interval mapping in the doubled haploid population derived from the cross Pingyuan $50 \times$ Mingxian 169

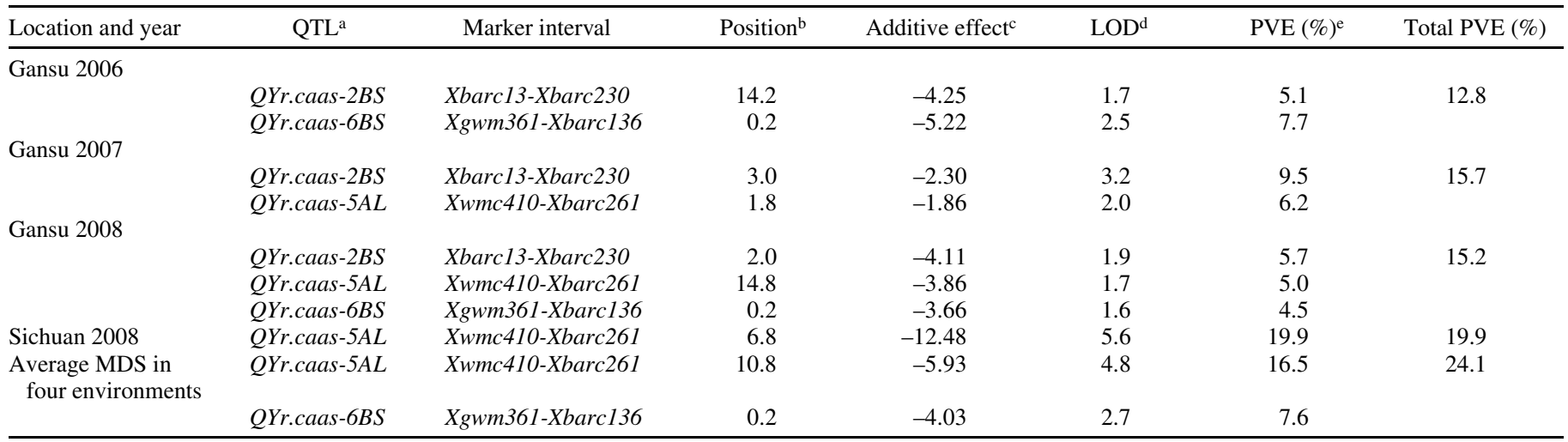

a QTL that overlap in the one-log support confidence intervals were assigned the same symbol.

${ }^{\mathrm{b}}$ Peak position in centimorgans from the first interval marker.

${ }^{c}$ Additive effect of resistance allele.

${ }^{\mathrm{d}}$ Logarithm of odds (LOD) score.

e PVE is the percentages of phenotypic variance explained by individual QTL. 
phenotypic variance in Gansu 2007 and 2008, Sichuan 2008, and the mean MDS, respectively, with additive effects ranging from -12.48 to -1.86 . QYr.caas- $6 B S$, on the short arm of chromosome 6B, was flanked by Xgwm361 and Xbarc136, and explained from 4.5 to $7.7 \%$ of the phenotypic variance in two environments as well as the mean data over all four environments. All QTL were from Pingyuan 50.

\section{DISCUSSION}

Pingyuan 50 was an old Chinese landrace wheat cultivar with several desirable traits including durable resistance to stripe rust in addition to its relative high yield potential, strong stem, and broad adaptation (33). Yuan et al. (32) reported that Pingyuan 50 was resistant to eight Chinese races of $P$. striiformis $\mathrm{f}$. $\mathrm{sp}$. tritici at the adult-plant stage, and demonstrated quantitative inheritance of APR to stripe rust, which is in agreement with the present study where three QTL effective in two or more environments were detected.

Ramburan et al. (20) identified a QTL for stripe rust response flanked by markers Xpsp3030 and $s 16 m 40 A$ on chromosome 2BS in a DH population of Kariega $\times$ Avocet. Guo et al. (6) found a QTL at a similar position, located in the marker interval Xgwm148-Xbarc167 in an $\mathrm{F}_{2: 3}$ population of Luke $\times$ Aquileja. Based on the wheat consensus map of Somers et al. (24), these two QTL and QYr.caas-2BS may involve the same or closely linked loci. QYr.caas-2BS was identified in Gansu from 2006 to 2008. It showed a relatively stable genetic effect against stripe rust across different years. The SSR marker Xbarc55 closely

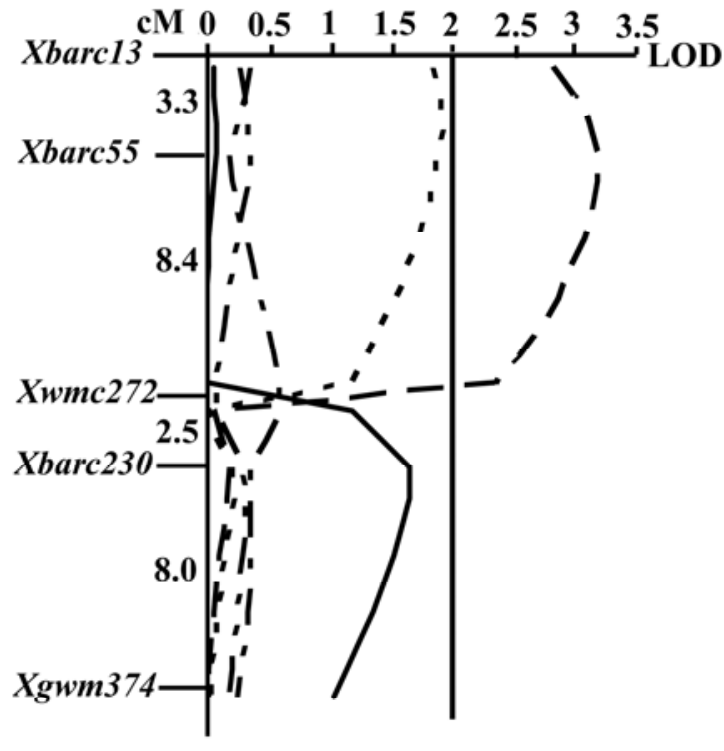

2BS

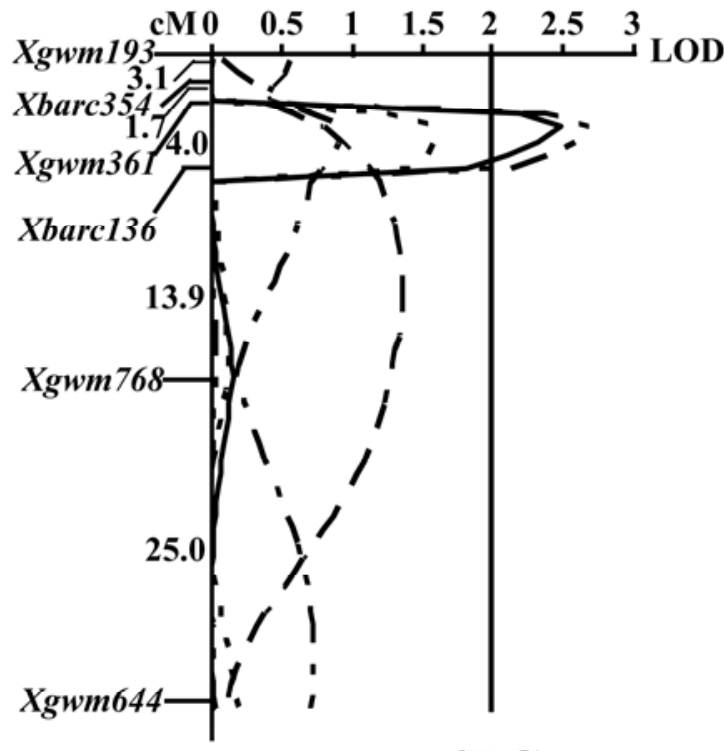

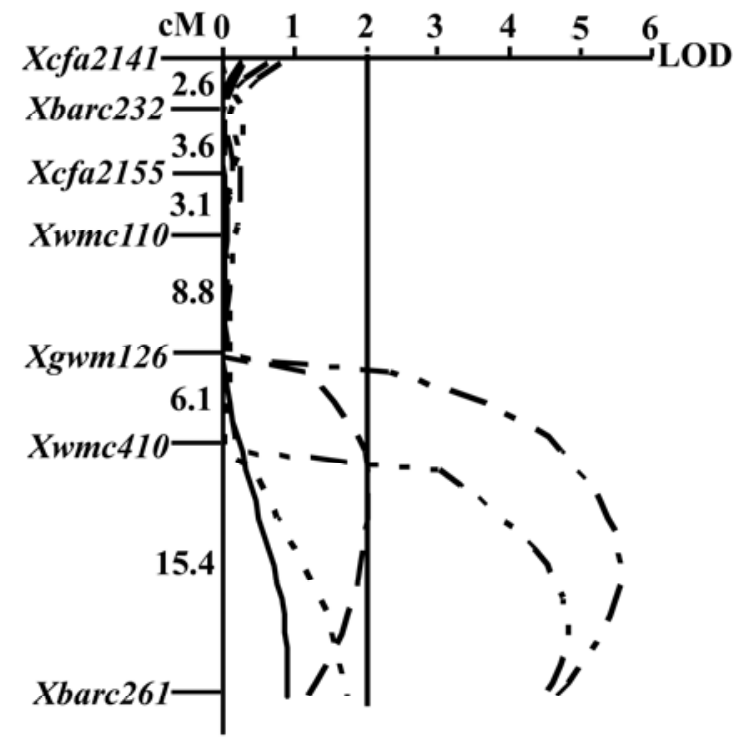

5AL

\section{BS}

Fig. 2. Logarithm of odds (LOD) contours obtained by inclusive composite interval mapping for quantitative traits loci on chromosomes $2 \mathrm{BS}$, $5 \mathrm{AL}$, and $6 \mathrm{BS}$ that reduce stripe rust severity in Pingyuan 50 × Mingxian 169 doubled haploid population. Gansu 2006, Gansu 2007, and Gansu 2008, maximum disease severity (MDS) in Gansu, 2006, 2007, and 2008, respectively. Sichuan 2008, maximum disease severity (MDS) in Sichuan 2008. Average, mean MDS over four environments. The LOD thresholds of 2.0 are indicated by a line. 
linked to this QTL could be used for improving wheat stripe rust resistance in breeding programs.

Boukhatem et al. (2) detected a QTL at the marker interval $X f b b 209-5 A-X a b g 391-5 A$ in the ITMI recombinant line population derived from Opata $85 \times$ Synthetic. Based on the wheat consensus map (24), this QTL is about $26.0 \mathrm{cM}$ distal to QYr.caas-5AL, and therefore they should be different. Chhuneja et al. (3) mapped a QTL on chromosome 5AL in the diploid A genome population $T$. monococcum (accession Pau14087) $\times T$. boeoticum (Pau5088), and this QTL was flanked by markers Xbarc151 and Xcfd12, that are an estimated $68.7 \mathrm{cM}$ from QYr.caas-5AL (24). One race-specific resistance gene has been mapped on chromosome 5AL, i.e., Yr34, in the Australian advanced breeding line WAWHT2046 (1). It produced an intermediate seedling infection type on $P$. striiformis $\mathrm{f}$. $\mathrm{sp}$. tritici pt134 E16A+, but was not against $110 \mathrm{E} 143 \mathrm{~A}+(1)$, indicating a race-specific nature. Its position is about $34 \mathrm{cM}$ from QYr.caas$5 A L$ based on the consensus map (24). In contrast, QYr.caas-5AL has been resistant to all the prevalent Chinese races at adult plants for more than 50 years, exhibiting a race nonspecific nature $(32,33)$. Therefore, QYr.caas-5AL appears to be a new gene conferring APR to stripe rust. It was detected with high LOD scores in three environments and in the mean MDS analysis. Selection for the SSR markers Xwmc410 and Xbarc261 closely linked to QYr.caas-5AL could be undertaken to improve APR to stripe rust in wheat.

Santra et al. (22) found a QTL for high temperature adult-plant resistance in cultivar Stephens. It was flanked by SSR markers Xbarc101 and Xbarc136 and located at a similar position to QYr.caas-6BS. Suenaga et al. (27) identified a QTL in cultivar Oligoculm, closely linked to microsatellite marker Xgwm935 and with an estimated map distance about $2 \mathrm{cM}$ from QYr.caas- $6 B S$ based on the consensus map (24). Another QTL possibly at this locus was found by Christiansen et al. (4) in cultivars Solist and Kris. It was flanked by SSR markers $\mathrm{Xgwm} 518$ and Xwmc105b and was estimated to be about $2.5 \mathrm{cM}$ away from QYr.caas- $6 B S$ (24). Thus, these QTL are at the same or closely linked loci. Uauy et al. (28) identified APR gene Yr36 on chromosome 6BS in DIC lines from $T$. turgidum sp. dicoccoides. Yr36 was completely linked to Xbarc101, and within $2 \mathrm{cM}$ interval defined by PCRbased markers Xucw71 and Xbarc136. However, QYr.caas-6BS came from T. aestivum, and thus, QYr.caas-6BS and Yr36 should be different stripe rust resistance genes. Although QYr.caas-6BS was identified only with relatively low LOD scores (1.6 to 2.7) and small effects, it was relatively stable across two environments and the averaged MDS. Reports of QTL located at the same position in previous studies add credence to this conclusion.

Compared with major race-specific resistance genes, APR genes have relatively small effects on stripe rust response and it is necessary to incorporate three or more such genes in a single genotype to obtain acceptably high levels of resistance (23). Molecular markers are obviously extremely useful for developing or maintaining such gene combinations in segregating breeding populations. Moreover, molecular markers are essential to detect such APR genes when major seedling resistance genes are present (28). We previously identified five QTL for stripe rust resistance in Italian wheat cultivars Libellula and Strampelli (17), and have found three additional QTL in the present study. In order to validate the potential use of these genes we have made many crosses between these resistant parents and Chinese commercial cultivars with good agronomical traits. Molecular markers identified in this and earlier research will facilitate the selection of combined APR gene combinations among the segregating progeny.

\section{ACKNOWLEDGMENTS}

We are very grateful to R. A. McIntosh, Plant Breeding Institute, University of Sydney, for his critical review of this manuscript. This study was supported by the National Science Foundation of China (30810214 and 30671294) and International Collaboration Project from the Ministry of Agriculture (2006-G2).

\section{LITERATURE CITED}

1. Bariana, H. S., Parry, N., Barclay, I. R., Loughman, R., McLean, R. J., Shankar, M., Wilson, R. E., Willey, N. J., and Francki, M. 2006. Identification and characterization of stripe rust resistance gene $\mathrm{Yr} 34$ in common wheat. Theor. Appl. Genet. 112:1143-1148.

2. Boukhatem, N., Baret, P. V., Mingeot, D., and Jacquemin, J. M. 2002. Quantitative trait loci for resistance against yellow rust in two wheatderived recombinant inbred line populations. Theor. Appl. Genet. 104:111-118.

3. Chhuneja, P., Kaur, S., Garg, T., Ghai, M., Kaur, S., Prashar, M., Bains, N. S., Goel, R. K., Keller, B., Dhaliwal, H. S., and Singh, K. 2008. Mapping of adult plant stripe rust resistance genes in diploid A genome wheat species and their transfer to bread wheat. Theor. Appl. Genet. 116:313-324.

4. Christiansen, M. J., Feenstra, B., Skovgaard, I. M., and Andersen, S. B. 2006. Genetic analysis of resistance to yellow rust in hexaploid wheat using a mixture model for multiple crosses. Theor. Appl. Genet. 112:581591.

5. Dong, Y. C., and Zheng, D. S. 2000. Wheat Genetic Resources in China. (In Chinese). China Agriculture Press, Beijing.

6. Guo, Q., Zhang, Z. J., Xu, Y. B., Li, G. H., Feng, J., and Zhou, Y. 2008. Quantitative trait loci for high-temperature adult-plant and slow-rusting resistance to Puccinia striiformis f. sp. tritici in wheat cultivars. Phytopathology 98:803-809.

7. Gupta, P. K., Rustgi, S., Sharma, S., Singh, R., Kumar, N., and Balyan, H. S. 2003. Transferable EST-SSR markers for the study of polymorphism and genetic diversity in bread wheat. Mol. Genet. Genet. 270:315-323.

8. Guyomarc'h, H., Sourdille, P., Edwards, K. J., and Bernard, M. 2002. Studies of the transferability of microsatellites derived from Triticum tauschii to hexaploid wheat and to diploid related species using amplification, hybridization and sequence comparisons. Theor. Appl. Genet. 105:736-744.

9. Johnson, R., and Taylor, A. J. 1972. Isolates of Puccinia striiformis collected in England from wheat varieties Maris Beacon and Joss Cambier. Nature 238:105-106.

10. Kosambi, D. D. 1944. The estimation of map distance from recombination values. Annu. Eugen. 12:172-175.

11. Laurie, D. A., and Bennett, M. D. 1988. The production of haploid wheat plants from wheat $\times$ maize crosses. Theor. Appl. Genet. 76:393-397.

12. Li, H. H., Ye, G. Y., and Wang, J. K. 2007. A modified algorithm for the improvement of composite interval mapping. Genetics 175:361-374.

13. Li, Z. F., Xia, X. C., Zhou, C. X., Niu, Y. C., He, Z. H., Zhang, Y., Li, G. Q., Wan, A. M., Wang, D. S., Chen, X. M., Lu, Q. L., and Singh, R. P. 2006. Seedling and slow rusting resistance to stripe rust in Chinese common wheats. Plant Dis. 90:1302-1312.

14. Lin, F., and Chen, X. M. 2008. Quantitative trait loci for non-racespecific, high-temperature adult-plant resistance to stripe rust in wheat cultivar Express. Theor. Appl. Genet. 116:797-806.

15. Lincoln, S., Daly, M., and Lander, E. 1992. Constructing genetic maps with Mapmaker/EXP3.0. Whitehead Institute Tech. Rep, 3rd ed. Whitehead Institute, Cambridge.

16. Line, R. F., and Chen, X. M. 1995. Success in breeding for and managing durable resistance to wheat rusts. Plant Dis. 79:1254-1255.

17. Lu, Y. M., Lan, C. X., Liang, S. S., Zhou, X. C., Liu, D., Zhou, G., Lu, L. Q., Jing, J. X., Wang, M. N., Xia, X. C., and He, Z. H. 2009. QTL mapping for adult-plant resistance to stripe rust in Italian common wheat cultivars Libellula and Strampelli. Theor. Appl. Genet. 119:1349-1359.

18. McIntosh, R. A., Dubcovsky, J., Rogers, W. J., Morris, C. F., Appels, R., and Xia, C. X. 2009. Catalogue of gene symbols for wheat: 2009 (suppl.). http://www.wheat.pw.usda.gov/GG2/pubs.shtml.

19. Peterson, R. F., Campbell, A. B., and Hannah, A. E. 1948. A diagrammatic scale for estimating rust intensity of leaves and stems of cereals. Can. J. Res. Sect. C. 26:496-500.

20. Ramburan, V. P., Pretorius, Z. A., Louw, J. H., Boyd, L. A., Smith, P. H., Boshoff, W. H. P., and Prins, R. 2004. A genetic analysis of adult plant resistance to stripe rust in the wheat cultivar Kariega. Theor. Appl. Genet. 108:1426-1433.

21. Röder, M. S., Korzun, V., Wendehake, K., Plaschke, J., Tixier, M. H., Leroy, P., and Ganal, M. W. 1998. A microsatellite map of wheat. Genetics 149:2007-2023.

22. Santra, D. K., Chen, X. M., Santra, M., Campbell, K. G., and Kidwell, K. K. 2008. Identification and mapping QTL for high-temperature adultplant resistance to stripe rust in winter wheat (Triticum aestivum L.) 
cultivar Stephens. Theor. Appl. Genet. 117:793-802.

23. Singh, R. P., Huerta-Espino, J., and Rajaram, S. 2000. Achieving nearimmunity to leaf and stripe rusts in wheat by combining slow rusting resistance genes. Acta. Phyto. Entom. Hung. 35:133-139.

24. Somers, D. J., Isaac, P., and Edwards, K. 2004. A high-density microsatellite consensus map for bread wheat (Triticum aestivum L.). Theor. Appl. Genet. 109:1105-1114.

25. Song, Q. J., Fickus, E. W., and Cregan, P. B. 2002. Characterization of trinucleotide SSR motifs in wheat. Theor. Appl. Genet. 104:286-293.

26. Sourdille, P., Singh, S., Cadalen, T., Brown-Guedira, G. L., Gay, G., Qi, L., Gill, B. S., Dufour, P., Murigneux, A., and Bernard, M. 2004. Microsatellite-based deletion bin system for the establishment of geneticphysical map relationships in wheat (Triticum aestivum L.). Funct. Int. Gen. 4:12-25.

27. Suenaga, K., Singh, R. P., Huerta-Espino, J., and William, H. M. 2003. Microsatellite markers for genes $\operatorname{Lr} 34 / \mathrm{Yr} 18$ and other quantitative trait loci for leaf rust and stripe rust resistance in bread wheat. Phytopathology 93:881-890.

28. Uauy, C., Brevis, J. C., Chen, X. M., Khan, I., Jackson, L., Chicaiza, O., Distelfeld, A., Fahima, T., and Dubcovsky, J. 2005. High-temperature adult-plant (HTAP) stripe rust resistance gene Yr36 from Triticum turgidum ssp. dicoccoides is closely linked to the grain protein content locus $G p c-B 1$. Theor. Appl. Genet. 112:97-105.

29. Wan, A. M., Zhao, Z. H., Chen, X. M., He, Z. H., Jin, S. L., Jia, Q. Z., Yao, G., Yang, J. X., Wang, B. T., Li, G. B., Bi, Y. Q., and Yuan, Z. Y. 2004. Wheat stripe rust epidemic and virulence of Puccinia striiformis $\mathrm{f}$. sp. tritici in China in 2002. Plant Dis. 88:896-904.

30. William, H. M., Singh, R. P., Huerta-Espino, J., Palacios, G., and Suenaga, K. 2006. Characterization of genetic loci conferring adult plant resistance to leaf rust and stripe rust in spring wheat. Genome 49:977990.

31. Yahyaoui, A. H., Hovmøler, M. S., Ezzahiri, B., Jahoor, A., Maatougui, M. H., and Wolday, A. 2004. Survey of barley and wheat diseases in the central highlands of Eritrea. Phytopatholgy 43:39-43.

32. Yuan, W. H., Zhang, Z. J., and Zeng, S. M. 1994. Inheritance of durable resistance to stripe rust in wheat variety Pingyuan 50. Acta Phyto. Sin. 24(1):39-42. (In Chinese with English abstract).

33. Zeng, S. M., Wang, F. Y., Wu, X. Y., Zhang, W. Q., Wang, J. Q., Song, W. Z., and Wang, S. Y. 1979. Analysis of horizontal resistance against stripe rust in wheat. Plant Protect 6(1):1-10. (In Chinese). 